\title{
Geophysical characteristics and distribution prediction of the Permian karst reservoirs in the Hebaochang area, South Sichuan Basin
}

\author{
QINGCHUN JIANG ${ }^{1}$ HUA JIANG $^{1}$ CONGSHENG BIAN $^{1}$ \\ 1PetroChina Research Institute of Petroleum Exploration and Development, Beijing 100083, China
}

Previous studies on the karst reservoirs of Maokou Fm, Middle Permian in the Southern Sichuan Basin mainly concentrate on sedimentary facies, reservoir characteristics and controlling factors, gas accumulation patterns and gas-source correlation, and well location deployment is carried out with faults as the focus. Due to the limitation of seismic data quality and other factors, this set of reservoirs are less analyzed qualitatively and predicted quantitatively. In this paper, the seismic response characteristics of high yield gas wells in karst reservoirs were analyzed based on the above mentioned traditional research achievements, combined with the 3D seismic data in the Hebaochang area of the Southern Sichuan Basin. Then, qualitative analysis, quantitative prediction and hydrocarbon detection were carried out on the karst reservoirs of Maokou Fm, Middle Permian by means of forward modeling, amplitude attribute analysis, hydrocarbon detection and other methods. Finally, the favorable areas for the development of karst reservoirs were predicted. And the following research results were obtained. First, the Maokou karst reservoirs in the Hebaochang area are vertically stratified and high yield intervals are mainly developed at the upper sections within $50 \mathrm{~m}$ from the top of Maokou Fm. The events of high yield intervals, i.e., top Maokou Fm and the lower part, present the seismic response characteristics of "weak peak \& weak and wide valley". The quantitative prediction of amplitude index attribute and the hydrocarbon detection results show that both of them are coincident and there are 3 high-quality reservoir development zones in this area. And their coincidence with the actual wells is up to $80 \%$. It is concluded that besides the favorable reservoir areas developed along the faults in the Hebaochang area, 3 favorable karst reservoir areas are developed far from the fault belt, the total area of which is $79 \mathrm{~km} 2$. The research results are of great significance to guiding the oil \& gas exploration and well location deployment in the structural slope and syncline areas far from faults. 